\title{
Generalizations of Heisenberg uncertainty relation
}

\author{
D.A. Trifonov \\ Institute for nuclear research, \\ 72 Tzarigradsko chaussee, 1784, Sofia
}

quant-ph/0112028

v.2: Mar 6, 2002

\begin{abstract}
A survey on the generalizations of Heisenberg uncertainty relation and a general scheme for their entangled extensions to several states and observables is presented. The scheme is illustrated on the examples of one and two states and canonical quantum observables, and spin and quasi-spin components. Several new uncertainty relations are displayed. PACS 0365H, 4250D, 0220
\end{abstract}

\section{Introduction}

The uncertainty (indeterminacy) principle exhibits a fundamental manner in which the quantum description of nature departs from the classical one. It was introduced in 1927 by Heisenberg [1] who demonstrated the impossibility of simultaneous precise measurement of the canonical quantum observables $x$ and $p_{x}$ (the coordinate and the momentum) by positing an approximate relation $\delta p_{x} \delta x \sim \hbar$. Soon after the Heisenberg paper appeared Kennard proved [2] the inequality (furthermore we use the dimensionless $p$ and $q,[p, q]=$ $-i)$

$$
(\Delta p)^{2}(\Delta q)^{2} \geq 1 / 4
$$

where $(\Delta p)^{2}$ and $(\Delta q)^{2}$ are the variances (dispersions) of $p$ and $q$, defined for any observable $X$ by $(\Delta X)^{2}:=\left\langle(X-\langle X\rangle)^{2}\right\rangle$. The inequality (11) became known as the Heisenberg uncertainty relation (Heisenberg UR) for the two canonical observables.

Generalization of inequality (1) to the case of arbitrary two observables (Hermitian operators $X$ and $Y$ ) was made by H. Robertson in 1929 [3],

$$
(\Delta X)^{2}(\Delta Y)^{2} \geq \frac{1}{4}|\langle[X, Y]\rangle|^{2} .
$$

Robertson inequality (2) became known again as the Heisenberg UR. However we prefer, in view of the Robertson contribution, to call it Heisenberg-Robertson (H-R) UR. This inequality (or its particular case (1i) became an irrevocable part of every textbook on quantum mechanics and it is regarded as a rigorous formulation of the Heisenberg indeterminacy principle.

The aim of the present paper is to consider symmetry properties of the H-R UR (section 2) and its natural invariant generalizations to several observables and several

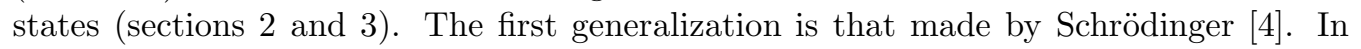
section 2 we briefly analyze the invariance properties of H-R UR and Schrödinger UR. Three basic UR's for $n$ observables and 2 state are displayed in subsection 3.2. In section 
3.3 a general scheme for construction of UR's for $n$ observables and $m$ states is provided. The relation of the conventional inequalities to the sets of the widely used canonical coherent states [5] and squeezed states [6, , 7] is also reminded.

\section{Invariant generalizations of Heisenberg-Robertson relation}

The conventional UR's (11) and (2) suffers from two deficiencies. The first one is that they are not form-invariant under the linear transformations of operators. In particular (iD) is not invariant under linear canonical transformations: if (1) is minimized in a state $|\psi\rangle$, then the canonical transformation (rotation on angle $\theta$ in phase space)

$$
p^{\prime}=p \cos \theta+q \sin \theta, \quad q^{\prime}=-p \sin \theta+q \cos \theta,
$$

violates the equality in (1). So it makes sense to look for other uncertainty inequalities, which are invariant under rotation (3). At the "level" of two second moments of $p$ and $q$ such inequality is

$$
(\Delta p)^{2}+(\Delta q)^{2} \geq 1
$$

This inequality is less precise than (1): the minimization of (14) entails the equality in (11), the inverse being untrue. For two arbitrary observables (14) takes the form

$$
(\Delta X)^{2}+(\Delta Y)^{2} \geq|\langle[X, Y]\rangle| .
$$

If $X$ and $Y$ are elements of a Lie algebra $L$, then their "linear canonical transformations" are the automorphisms in $L$.

The second point is that for two observables there are three second order statistical moments - the variances of each observable and their covariance, while only the first two ones are taken into account in (2). This fact was first noted by Schrödinger 4, who derived (using Schwartz inequality) the more general inequality

$$
(\Delta X)^{2}(\Delta Y)^{2}-(\Delta X Y)^{2} \geq \frac{1}{4}|\langle[X, Y]\rangle|^{2}
$$

where $\Delta X Y$ denotes the covariance of $X$ and $Y, \Delta X Y=\langle X Y+Y X\rangle / 2-\langle X\rangle\langle Y\rangle$. In the classical probability theory the vanishing covariance is a necessary (but not sufficient) condition for the statistical independence of two random quantities.

In the case of coordinate and momentum observables the relation (6) takes the shorter form of

$$
(\Delta q)^{2}(\Delta p)^{2}-(\Delta q p)^{2} \geq 1 / 4
$$

Schrödinger inequality (6) is more general and more precise than that of HeisenbergRobertson, eq. (2): the former is reduced to the latter in states with vanishing covariance of $X$ and $Y$, and the equality in (2) entails the equality in (6), the inverse being untrue.

One can easily check that Schrödinger UR is invariant under all linear canonical transformations of $p$ and $q$, including the scale transformations. From the three inequalities (11), (伍) and (7) it is the Schrödinger one that is most precise and the most symmetric. The inequality (蛋 is the most unprecise one: the equality in it entails the equality in both (7) and (11).

The interest in Schrödinger relation has grown in the last two decades in connection with the description and experimental realization of the canonical squeezed states of the electromagnetic radiation [6, 7]. This family can be defined [8] as the unique set of states that minimize inequality (6). It was only recently realized [6], that the famous canonical coherent states (introduced in [5]) can be uniquely defined as states that minimize (4). 


\section{Generalizations to several observables and several states}

The uncertainty relations (11), (2) and (6) provide a quantitative limitations to the accuracy of measurement of two incompatible observables in one and the same state. Two natural questions related to inequalities (1) - (7), can be immediately formulated:

(a) are there nontrivial generalizations to the case of several observables and one state?

(b) are there nontrivial generalizations to the case of one or several observables and several states?

By "nontrivial generalizations" I mean uncertainty inequalities, which can not be represented as sums or products of those for two observables and one state. Such UR's could be called observable- or state- entangled.

\subsection{Robertson inequalities for $n$ observables}

The positive answer to the first of the above two questions was given by H. Robertson in 1934 [9], who generalized (2) and (6) to the case of $n$ observables. For $n$ Hermitian operators $X_{i}, i=1, \ldots, n$, Robertson established the inequality $\left(\vec{X}=\left(X_{1}, \ldots, X_{n}\right)\right)$

$$
\operatorname{det} \sigma(\vec{X}) \geq \operatorname{det} C(\vec{X})
$$

where $\sigma$ is the uncertainty matrix, and $C$ is a matrix of mean commutators of $X_{i}$, and $X_{j}$ :

$$
\sigma_{i j}=\frac{1}{2}\left\langle X_{i} X_{j}+X_{j} X_{i}\right\rangle-\left\langle X_{i}\right\rangle\left\langle X_{j}\right\rangle, C_{i j}=-\frac{i}{2}\left\langle\left[X_{i}, X_{j}\right]\right\rangle .
$$

The diagonal element $\sigma_{i i}$ is just the variance of $X_{i}$, while $\sigma_{i j}$ is the covariance of $X_{i}$ and $X_{j}$. At $n=2$ inequality (8) recovers (6).

Robertson UR (8) is form-invariant with respect to any nondegenerate linear real transformation of $n$ operators $X_{i}$, the equality being invariant. Indeed, let $X_{i}^{\prime}=\lambda_{i j} X_{j}$, where $\Lambda=\left(\lambda_{i j}\right)$ is non-degenerate. Then the uncertainty matrix $\sigma^{\prime}$ for $X_{i}^{\prime}$ in the same state is obtained as

$$
\sigma^{\prime}:=\sigma\left(\vec{X}^{\prime}\right)=\Lambda \sigma \Lambda^{T}
$$

where $\Lambda^{T}$ is transposed $\Lambda$. Similarly $C^{\prime}=\Lambda C \Lambda^{T}$. Then we see that the equality $\operatorname{det} \sigma^{\prime}=\operatorname{det} C^{\prime}$ follows from $\operatorname{det} \sigma=\operatorname{det} C$ and vice versa. Therefore (8) generalizes the full symmetry properties of Schrödinger relation (6) to the case of $n$ observables. Robertson also noted the generalization of the less precise inequality (2): from the Hadamard inequality and (8) he immediately derived the inequality (to be called Hadamard-Robertson UR)

$$
\left(\Delta X_{1}\right)^{2} \ldots\left(\Delta X_{n}\right)^{2} \geq \operatorname{det} C(\vec{X}) .
$$

Here I provide the generalization of the most unprecise inequality (5) to the case of $n$ arbitrary observables $X_{i}$ :

$$
\operatorname{Tr} \sigma(\vec{X})=\sum_{i}^{n}\left(\Delta X_{i}\right)^{2} \geq \frac{1}{n-1} \sum_{j>i}^{n}\left|\left\langle\left[X_{i}, X_{j}\right]\right\rangle\right| .
$$

This inequality holds for any $n$. For even $n, n=2 m$ it can be enhanced,

$$
\operatorname{Tr} \sigma(\vec{X}) \geq \sum_{\mu=1}^{m}\left|\left\langle\left[X_{\mu}, X_{m+\mu}\right]\right\rangle\right|,
$$

Note that $(8)$ is most precise and most symmetric: it is form-invariant under any nondegenerate linear transformation $\vec{X} \longrightarrow \vec{X}^{\prime}=\Lambda \vec{X}$. One can check that the most unprecise inequality (11) is invariant under orthogonal linear transformations of $X_{i}$. The 
intermediate precision inequality (10) is most unsymmetric: it is invariant under special scale transformations $X_{k} \rightarrow X_{k} / \alpha_{k}, X_{m+k} \rightarrow \alpha_{k} X_{m+k}$ only $(k=1, \ldots m, m \leq[n / 2])$.

The problem of minimization of Robertson relation (8) is considered in [11] (see also [7]). It is worth noting the result that the group-related coherent states with maximal symmetry (see [12] and references therein), the simplest examples of which are the spin and the quasi-spin coherent states, are the unique states that minimize Robertson inequality (8) for the Hermitian components of the Weyl lowering and raising operators. Therefore these states can be alternatively defined as Robertson minimum uncertainty states (called also Robertson intelligent states [11]) for several observables.

On the example of $2 m$ canonical observables $X_{i}=Q_{i}, Q_{\mu}=p_{\mu}, Q_{m+\mu}=q_{\mu}$, inequalities (8) and (10) and (12) read

$$
\begin{gathered}
\operatorname{det} \sigma(\vec{Q}) \geq \frac{1}{4^{m}} \\
\left(\Delta p_{1}\right)^{2}\left(\Delta q_{1}\right)^{2} \cdots\left(\Delta p_{m}\right)^{2}\left(\Delta q_{m}\right)^{2} \geq \frac{1}{4^{m}}, \\
\operatorname{Tr} \sigma(\vec{Q})=\sum_{\mu=1}^{m}\left[\left(\Delta p_{\mu}\right)^{2}+\left(\Delta q_{\mu}\right)^{2}\right] \geq m .
\end{gathered}
$$

The equality in (13) is reached in the multimode squeezed states [6, 7] (for this and other examples of states that minimize (8) with $n>2$ see refs. [11, 7]). The squeezed states with vanishing covariances of all $p_{\mu}$ and $q_{\nu}$ minimize (10), while (15) is minimized only in multimode canonical coherent states (in which one also has $\Delta p_{\mu} q_{\nu}=0$ ).

Let us note that $\operatorname{Tr} \sigma(\vec{Q})$ (and $\operatorname{Tr}(\sigma(\vec{Q}))^{k}$ as well) is invariant under orthogonal transformations $\vec{Q} \longrightarrow \Lambda \vec{Q}$, but not under symplectic ones. Symplectic transformations preserve the canonical commutation relations. In the one mode case, i.e. $m=1$, all orthogonal matrices are symplectic. Invariant under symplectic transformations is the quantity $\operatorname{Tr}(\sigma J)^{k}$. In ref. 10, 11 the following invariant inequalities were proved (please note that in ref. [10] factor $i$ is omitted)

$$
\operatorname{Tr}[i \sigma(\vec{Q}) J]^{2 k} \geq m / 2^{2 k-1} .
$$

At $m=1$ and $k=1$ Schrödinger UR (7) is recovered.

The $2 m$ canonical operators $Q_{i}$ are known to close the Heisenberg-Weyl Lie algebra. In the general case of $X_{i}$ closing any Lie algebra the right hand sides of the above UR's (8), (10) - (12) are, due to the commutation relations $\left[X_{i}, X_{k}\right]=c_{i k}^{j} X_{j}$, combinations of mean values of $X_{i}$. Let us note the case of $s u(2) \sim s o(3)$ and $s u(1,1) \sim s o(2,1)$ algebras. The three basic operators $X_{i}=J_{i}$ of $s u(2)$ are the spin operators, and the three generators $K_{i}$ of $s u(1,1)$ are known as quasi-spin operators:

$$
\left[J_{k}, J_{l}\right]=i \epsilon_{k l m} J_{m}, \quad\left[K_{1}, K_{2}\right]=-i K_{3},\left[K_{2}, K_{3}\right]=i K_{1},\left[K_{3}, K_{1}\right]=-i K_{2} .
$$

The inequality (11), applied to $J_{i}$ and $K_{i}$, tells us that the sum of fluctuations (variances) of spin or quasi-spin components is not less than the sum of their absolute mean values,

$$
\sum_{i=1}\left(\Delta J_{i}\right)^{2} \geq \frac{1}{2} \sum_{i=1}\left|\left\langle J_{i}\right\rangle\right|, \quad \sum_{i=1}\left(\Delta K_{i}\right)^{2} \geq \frac{1}{2} \sum_{i=1}\left|\left\langle K_{i}\right\rangle\right| .
$$

\subsection{Extension of Heisenberg and Schrödinger relations to two distinct states}

All the uncertainty relations so far considered in the literature (and the above (8)-(16) as well) have the form of inequalities between first and second (or higher) moments of the 
observables in one and the same state. Furthermore such uncertainty relations should be called conventional.

However it is clear that one can measure and compare the accuracy of measurement of observables in distinct states. Therefore it is reasonable to look for nontrivial inequalities between statistical moments of observables in two and several states. Such relation should be called state-extended or state-entangled. On the lowest level of two observables such extended inequalities can be obtained by somewhat elementary manipulations of the Heisenberg-Robertson and/or Schrödinger inequalities written for two distinct states. Here are the state-entangled extensions of the conventional relations (何, (1) and (6) for $p$ and $q$ to the case of two state $|\phi\rangle$ and $|\psi\rangle$,

$$
\begin{gathered}
\Delta_{\psi} q \Delta_{\phi} p+\Delta_{\phi} q \Delta_{\psi} p \geq 1, \\
\left(\Delta_{\psi} q\right)^{2}\left(\Delta_{\phi} p\right)^{2}+\left(\Delta_{\phi} q\right)^{2}\left(\Delta_{\psi} p\right)^{2} \geq \frac{1}{2}, \\
\left(\Delta_{\psi} q\right)^{2}\left(\Delta_{\phi} p\right)^{2}+\left(\Delta_{\phi} q\right)^{2}\left(\Delta_{\psi} p\right)^{2}-2\left|\Delta_{\psi} q p \Delta_{\phi} q p\right| \geq \frac{1}{2},
\end{gathered}
$$

where $\Delta_{\psi} X Y$ is the covariance of $X$ and $Y$ in the state $|\psi\rangle$. At $|\psi\rangle=|\phi\rangle$ the conventional inequalities (4), (1) and (6) are recovered. Note the symmetry under transpositions $p \leftrightarrow q$ and $|\psi\rangle \leftrightarrow|\phi\rangle$.

\subsection{General scheme for uncertainty relations}

A quite general scheme for construction of uncertainty relations for $n$ observables and $m$ states is provided by the following Lemma,

Lemma. If $H_{\mu}, \mu=1, \ldots, m$ are non-negative definite Hermitian $n \times n$ matrices, then the following inequalities hold

$$
\begin{gathered}
\mathcal{M}\left(i_{1}, \ldots, i_{r} ; \sum_{\mu} S_{\mu}\right) \geq \mathcal{M}\left(i_{1}, \ldots, i_{r} ; \sum_{\mu} A_{\mu}\right), \\
\mathcal{M}\left(i_{1}, \ldots, i_{r} ; \sum_{\mu} H_{\mu}\right) \geq \sum_{\mu} \mathcal{M}\left(i_{1}, \ldots, i_{r} ; H_{\mu}\right), \\
\operatorname{Tr} S_{\mu} \geq \frac{2}{n-1} \sum_{j>i}^{n}\left|\left(A_{\mu}\right)_{i j}\right| \quad \text { for any } n \\
\operatorname{Tr} S_{\mu} \geq \sum_{\nu=1}^{m}\left|\left(A_{\mu}\right)_{\nu, m+\nu}\right| \quad \text { for even } n=2 m,
\end{gathered}
$$

where $\mathcal{M}\left(i_{1}, \ldots, i_{r} ; B\right)$ is the principal minor of order $r \leq n$ of matrix $B$, and $S_{\mu}$ and $A_{\mu}$ are the symmetric and the antisymmetric part of $H_{\mu}: H_{\mu}=S_{\mu}+i A_{\mu}$.

The first two inequalities (20) and (21) are proved in the second paper of ref. 114 (Lemma 2 in 14]). The proof of the third matrix inequality of the Lemma can be performed along the following lines. We represent the trace of $S$ in the form $\operatorname{Tr} S=$ $(1 /(n-1)) \sum_{j>i}\left(S_{i i}+S_{j j}\right)$ and consider the $2 \times 2$ principal submatrices $S(i j)$ of $S$, $[S(i j)]_{11}=S_{i i},[S(i j)]_{12}=S_{i j}=[S(i, j)]_{21},[S(j j)]_{22}=S_{j j}$. These are symmetric and non-negative definite 14, 13]. Similarly we define $2 \times 2$ antisymmetric matrices $A(i j)$ and compose $H(i j)=S(i j)+i A(i j)$. Then we apply (20) to $H(i j)$ and use the Hadamard inequality to obtain $S_{i i}+S_{j j} \geq 2\left|A_{i j}\right|$, wherefrom the desired inequality (22) follows.

For even $n, n=2 m$, we put $\operatorname{Tr} S=\sum_{\nu=1}^{m}\left(S_{\nu \nu}+S_{\nu+m, \nu+m}\right)$ and in a similar way obtain inequality (23).

For brevity we shall call the above inequalities principal minor inequalities of type $(n, m)$ and order $r$. 
Since the characteristic coefficients $C_{r}^{(n)}(B)$ of order $r \leq n$ of any matrix $B$ are sums of the principal minors [13], the above inequalities (20) and (21) remain valid if one replaces $\mathcal{M}\left(i_{1}, \ldots, i_{r} ; B\right)$ with $C_{r}^{(n)}(B)$ for the corresponding $B$. (For example, in the left hand side of the first inequality (20) $B=\sum_{\mu} S_{\mu}$, and in the right hand side $\left.B=\sum_{\mu} A_{\mu}\right)$. The obtained inequalities for $C_{r}^{(n)}(B)$ are called characteristic. Note that $\mathcal{M}\left(i_{1}, \ldots, i_{n} ; B\right)=C_{n}^{(n)}(B)$ and $C_{n}^{(n)}(B)=\operatorname{det} B$, while $C_{n}^{(1)}(B)=\operatorname{Tr} B$.

By a suitable physical choice of matrices $H_{\mu}$ in matrix inequalities (20) - (23), and in the corresponding characteristic inequalities as well, one can obtain series of physical relations. If matrices $H_{\mu}$ are constructed by means of statistical moments of observables, then the obtained inequalities are UR's, which could naturally be called principal or characteristic UR's.

All UR's considered in the previous sections and subsections, including (16), can be casted in the forms (20) - (23). The conventional UR (16) is of the form (23) with $S=\left(i \sigma^{\prime}(\vec{Q}) J\right)^{2 k}$ and $A=\left(A_{i j}\right): A_{\mu \nu}=A_{m+\mu, m+\nu}=0, A_{\nu, m+\nu}=-A_{m+\nu, \nu}=1 / 2^{2 k-1}$, where $\sigma^{\prime}$ is the diagonal uncertainty matrix, obtained from $\sigma$ by means of a symplectic transformation $\vec{Q}^{\prime}=\Lambda \vec{Q}$ 11, 10]. Next we consider some illustrative examples of nonnegative matrices $H_{\mu}=H_{\mu}^{\dagger}, \mu=1, \ldots, m$, and the related new UR's.

Example 1. An illustrative physical choice of $H_{\mu}$ is the following $H_{\mu}=G_{\mu}\left(\vec{X} ; \psi_{\mu}\right)$, where

$$
G_{\mu ; i j}=\left\langle\left(X-\left\langle X_{i}\right\rangle\right) \psi_{\mu}\left|\left(X_{j}-\left\langle X_{j}\right\rangle\right)\right| \psi_{\mu}\right\rangle .
$$

Matrix $G_{\mu}$ can be recognized as Gram matrix for the transformed states $\left|\chi_{\mu i}\right\rangle=(X-$ $\left.\left\langle X_{i}\right\rangle\right)\left|\psi_{\mu}\right\rangle$. Its symmetric part is defined as the uncertainty matrix $\sigma$. For one state and $n$ operators $X_{i}$ the inequality (20) with $r=n$ and $H=G(\vec{X} ; \psi)$ coincides with Robertson inequality (8). For two states and 2 observables $p$ and $q$ the senior inequalities (20) and (21) coincide and (with $H_{\mu}=G\left(\vec{Q} ; \psi_{\mu}\right)$ ) reproduce (19); for arbitrary two $X$ and $Y$ (20) produces

$$
\begin{aligned}
& \left(\Delta_{\psi_{1}} X\right)^{2}\left(\Delta_{\psi_{2}} Y\right)^{2}+\left(\Delta_{\psi_{2}} X\right)^{2}\left(\Delta_{\psi_{1}} Y\right)^{2} \\
& \quad-2\left|\Delta_{\psi_{1}} X Y \Delta_{\psi_{2}} X Y\right| \geq \frac{1}{2}\left|\langle[X, Y]\rangle_{\psi_{1}}\langle[X, Y]\rangle_{\psi_{2}}\right| .
\end{aligned}
$$

This is a direct extension of conventional Schrödinger UR (6) to two distinct states.

Example 2. Another interesting family of uncertainty relations is provided by the choice $H_{\mu}={ }^{\prime} G_{\mu}\left(\vec{X} ; \psi_{\mu} ; k\right)$, where

$$
{ }^{\prime} G_{\mu ; i j}=\left\langle\left(X_{i}^{k}-\left\langle X_{i}\right\rangle^{k}\right) \psi_{\mu}\left|\left(X_{j}^{k}-\left\langle X_{j}\right\rangle^{k}\right)\right| \psi_{\mu}\right\rangle,
$$

where $X_{j}^{k}$ is the $k$-th power of $X_{j}$. Matrix ' $G_{\mu}$ can be recognized as Gram matrix for the transformed states $\left|{ }^{\prime} \chi_{\mu, i}\right\rangle=\left(X_{i}^{k}-\left\langle X_{i}\right\rangle^{k}\right)\left|\psi_{\mu}\right\rangle$. The diagonal elements of matrix ${ }^{\prime} G_{\mu}$ are nothing but the $k$-order statistical moments of $X_{i}$. Thus the resulting principal inequalities are uncertainty relations for higher statistical moments of $n$ observables in $m$ states.

Gram matrix $G$ for any $n$ vectors $\left|\chi_{\mu}\right\rangle=a_{\mu k}\left(X ; \psi_{k}\right)\left|\psi_{k}\right\rangle\left(a_{\mu k}\left(X, \psi_{k}\right)\right.$ being combinations of operators $X_{i}$ and their moments in $\left.\left|\psi_{k}\right\rangle\right)$ will produce some UR [14. Note that for one state $G=\langle\chi \mid \chi\rangle$ with $\operatorname{det} G=\langle\chi \mid \chi\rangle \geq 0$. For example, take the combination 15 $|\chi\rangle=|\phi\rangle-|\psi\rangle\langle\psi \mid \phi\rangle-\left|\psi_{X}\right\rangle\left\langle\psi_{X} \mid \phi\right\rangle$, where $\left|\psi_{X}\right\rangle=\left(X-\langle X\rangle_{\psi}\right)|\psi\rangle / \Delta_{\psi} X$. Now $\operatorname{det} G \geq 0$ immediately produces the inequality

$$
\Delta_{\psi} X \geq\left|\left\langle\phi\left|\left(X-\langle X\rangle_{\psi}\right)\right| \psi\right\rangle\right|^{2} / \sqrt{1-|\langle\phi \mid \psi\rangle|^{2}} \equiv f[X, \psi, \phi] .
$$


Similarly $\Delta_{\phi} X=f[X, \phi, \psi]$ ( $\psi$ and $\phi$ interganged $)$ and

$$
\Delta_{\psi} X \Delta_{\phi} X \geq f[X, \psi, \phi] f[X, \phi, \psi] \geq 0 .
$$

\section{Concluding remarks}

The presented scheme for construction of uncertainty relations is quite general, since it is based on abstract matrix inequalities (20) - (23). Any non-negative Hermitian matrices $H_{\mu}$ involving (second or higher) statistical moments of observables, both for quantum and classical stochastic observables, can be used in this scheme to produce a hierarchy of uncertainty relations of types $(n, m)$. We have shown that the basic quantity for the uncertainty relations in quantum physics is the Gram matrix. Its symmetric part can be regarded as a generalization of the uncertainty matrix $\sigma(\vec{X})$. This is most clear for Gram matrix of the form (24). This definition of $\sigma(\vec{X})$ persists for the states that are outside the domain of the product $X_{i} X_{j}$, and is valid for mixed states as well.

\section{References}

[1] W. Heisenberg, Z. für Phys. 43 (1927) 172-198.

[2] E.H. Kennard, Z. für Phys. 44 (1927) 326-352.

[3] H.P. Robertson, Phys. Rev. 34 (1929) 163-164.

[4] E. Schrödinger, Sitz. Preus. Acad. Wiss. (Phys.-Math. Klasse), 19 (1930) 296.

[5] R.J. Glauber, Phys. Rev. 131 (1963) 2766; J.R. Klauder, J. Math. Phys. 4 (1963) 1055; ibid, 1058.

[6] R. Loudon and P. Knight, J. Mod. Opt. 34 (1987) 709-759; D.N. Klyshko, Uspehi Fiz. Nauk 166 (1996) 613-638.

[7] D.A. Trifonov, JOSA A 17 (2000) 2486.

[8] D.A. Trifonov, J. Math. Phys. 34 (1993) 100.

[9] H.P. Robertson, Phys. Rev. 46 (1934) 794.

[10] E.C.G. Sudarshan, C.B. Chiu, and G. Bhamathi, Phys. Rev. A 52 (1995) 43.

[11] D.A. Trifonov, J. Phys. A 30 (1997) 5941.

[12] J.R. Klauder and B.-S. Skagerstam, Coherent States - Applications in Physics and Mathematical Physics (W. Scientific, Singapure, 1985).

[13] F.R. Gantmaher, Teoria matrits (Nauka, Moscow, 1975).

[14] D.A. Trifonov, J. Phys. A 33 (2000) L296; J. Phys. A 34 (2001) L75.

[15] G.N. Fleming, e-print physics/0106077. 